This journal is the official publication of Bangladesh Society of Physiologists (BSP)

Web URL: www.banglajol.info/index.php/JBSP

Abstracted /indexed in Index Copernicus, Director of Open Access Journal, HINARI Index Medicus for South East Asia Region, Google Scholar, 12OR, infobse index, Open J gate, Cite factor, Scientific indexing services

pISSN-1983-1213; e-ISSN-2219-7508

\title{
Article
}

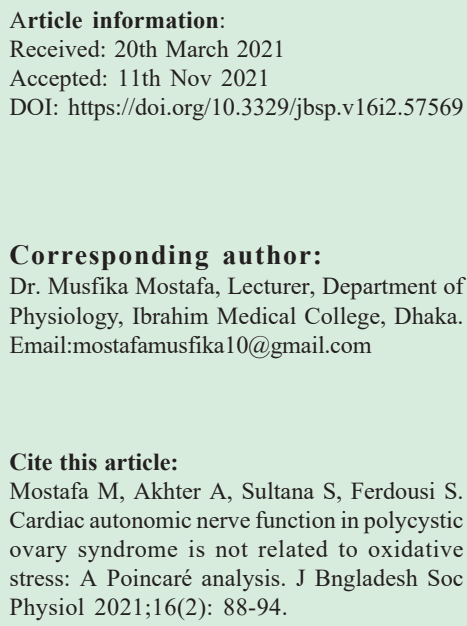

This article is open access licensed under CC BY NC SA which allows readers copy, distribute, display, and perform the work and make derivative works based on it only for noncommercial purposes.

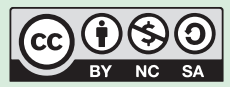

\section{Cardiac autonomic nerve function in polycystic ovary syndrome is not related to oxidative stress: A Poincaré analysis of heart rate variability}

\author{
Musfika Mostafa $^{1}$, Ayesha Akhter ${ }^{2}$, Shamima Sultana ${ }^{3}$, \\ Sultana Ferdousi ${ }^{3}$ \\ 1. Department of Physiology, Ibrahim Medical College, Dhaka \\ 2. Department of Physiology, Shaheed Monsur Ali Medical College, Dhaka \\ 3. Department of Physiology, Bangabandhu Sheikh Mujib Medical \\ University, Dhaka
}

\section{Abstract}

Background: Polycystic ovary syndrome (PCOS) is a well recognized reproductive hormone disorder. It is often associated with dysautonomia and increased oxidative stress Objective: To assess the relationship between cardiac autonomic nerve function (CANF) and oxidative stress in patients with PCOS. Methods: This cross sectional study enrolled 30 newly diagnosed PCOS patients 20-35 years of age and 30 apparently healthy, regularly menstruating women of similar age and BMI. The patients were enrolled from outpatient Department of Endocrinology, BSMMU, Dhaka. CANF was assessed by poincare analysis of RR interval, a non linear method of Heart Rate Variability (HRV) analysis. HRV data of all subjects were recorded by a digital data acquisition device, Power lab 8/35, AD instruments, Australia, with lab chart software analysis. Oxidative stress was assessed by plasma catalase and plasma Malondialdehyde (MDA) levels. For statistical analysis unpaired " $t$ " test and Pearson's correlation test were used. Results: In this study, resting pulse rate, systolic blood pressure(SBP), diastolic blood pressure(DBP) were significantly higher $(\mathrm{p}<0.001, \mathrm{p}<0.01, \mathrm{p}<0.01$ respectively) and standard deviation of the short term RR interval variability (SD1), ratio of short term and long term variability (SD1/SD2) ratio were 
significantly lower $(\mathrm{p}<0.001)$ in PCOS than those of healthy controls. In addition, plasma catalase levels was significantly lower $(\mathrm{p}<0.01)$ and plasma MDA levels was significantly higher $(p<0.001)$ in PCOS patients compared to controls. On correlation analysis, SD1 showed negative correlation with plasma catalase and plasma MDA levels $(\mathrm{p}>0.05)$ in PCOS patients. In addition $\mathrm{SD} 1 / \mathrm{SD} 2$ ratio showed positive correlation with plasma catalase and negative correlation with plasma MDA levels $(p>0.05)$ in these group of patients. But all these correlations were statistically not significant. Conclusion: The present study reveals association of autonomic dysfunction and higher oxidative stress with PCOS but it failed to show evidence for any interrelationship between them.

Key words: Heart rate variability, SD1,SD2, polycystic ovary syndrome, MDA, catalase.

Introduction

$\mathbf{P}$ olycystic ovary syndrome (PCOS) is a well recognized condition of reproductive hormone disorder. ${ }^{1}$ PCOS is usually associated with multiple cysts in ovaries and infertility in large number of patients. ${ }^{2}$

The prevalence rate of obesity or overweight in the PCOS population is about $50 \%$ and most of them have android phenotype which is partly responsible for insulin resistance and consequent hyperinsulinemia. ${ }^{3}$ Obesity and hyperinsulinaemia has long been known to cause derangement in autonomic functions associated with chronic sympathetic over activity and decreased vagal cardiac modulation. ${ }^{4-7}$

.Heart rate variability (HRV) analysis is a useful noninvasive tool widely used for measuring CANF. Among different methods of HRV analysis, Poincare plot analysis (PPA) is more sensitive and easier technique in nonlinear method. ${ }^{8} \mathrm{CANF}$ is affected in PCOS with sympathetic hyperactive and hypo parasympathetic ${ }^{6-7}$ or there may be no change in CANF. 5,9

Oxidative stress represents imbalance between body oxidant and antioxidant system ${ }^{10}$ increased oxidative stress has been reported in PCOS patients. ${ }^{11-13}$ lipid peroxidation product Malonaldehyde (MDA) is an oxidant and serum catalase as an antioxidant are important marker for oxidative stress. PCOS patients are at increased risk of cardiovascular disease (CVD) due to the associated insulin resistance, hypertension, central obesity, and dyslipidemia. $^{14-15}$

Several previous studies investigated HRV in PCOS by time domain and frequency domain method but HRV data by Poincare analysis (non linear method) is scarce. ${ }^{16}$ Altered HRV and higher oxidative challenges have been reported in PCOS but the correlation of Poincare data of HRV with oxidative stress has not been published. Therefore, this study has been designed to investigate the relationship between Poincare analysis of HRV and oxidant and antioxidant level in patients with PCOS.

\section{Methods \\ Setting \& study participants}

In this cross sectional study carried out in the Department of Physiology of BSMMU, Dhaka, from 2018 to 2019, 30 newly diagnosed PCOS 
patients, aged 20-35 years were enrolled from the outpatient Department of Endocrinology, BSMMU by purposive sampling. Similar age and BMI apparently healthy women with regular menstrual cycles (early follicular phase) were taken as controls. Sample size was calculated by a statistical formula based on effect size in published results by similar article.

\section{Exclusion criteria}

All the subjects were free from cardiac disease, respiratory disease, renal disease, diabetes mellitus and thyroid disorders. Women, who were pregnant or lactating or under treatment for infertility were excluded from this study.

\section{Procedure}

The protocol of this study was approved by the Institutional Review Board of BSMMU. After briefing about the study, informed written consent was taken from each subject. Detail family, medical and dietary history was recorded in a preformed data schedule and thorough physical examination was done. Resting pulse rate, $\mathrm{BP}$, height, weight were measured and BMI was calculated. Then $7 \mathrm{ml}$ of venous blood was serum SGPT, serum TSH and serum creatinine in the laboratory of Department of Biochemistry and Molecular Biology by autoanalyzer and plasma catalase and plasma MDA in the Department of Physiology by Catalase Assay Kit by colorimetric method using Quantiamate RM2012 and by MDA- ELISA kit using AMP Platos R II Microplate Reader. (Elabscience Biotecnology Inc 2018).

Then the subjects were advised to follow some instructions to prepare for HRV. They were advised to finish their meal by 9:00 pm on the previous night of HRV test day, avoid any type of stress and not to take any sedative or hypnotic medication. They were requested to take a light breakfast without tea and coffee and to attend collected for estimation of fasting plasma glucose,

the autonomic nerve function test laboratory in the Department of Physiology, BSMMU between 8:00 am to 9:00am on the test day. The subject was advised to take rest for 15-20 minutes in a controlled laboratory environment. During this period she was not allowed to talk, eat or drink, to perform physical or mental activity or sleep. ECG was recorded on lead II for 5 minutes by data acquisition device Power Lab 8/35 (AD Instrument, Australia). HRV recording was analyzed by Lab chart software.

\section{Statistical analysis}

Data were expressed as Mean \pm SD. Statistical analysis was done using SPSS version 16. Independent sample ' $t$ ' test and pearson correlation coefficient test analysis were done, $p$ value of $<0.05$ was considered as statistically significant.

\section{Results}

Anthropometric data of all subjects are given in Table I. Both groups were similar for age, BMI and waist hip ratio. Mean resting pulse rate $(\mathrm{p}<0.001)$, SBP and DBP $(\mathrm{p}<0.01)$ were significantly higher and mean values of SD1 and SD1/SD2 were significantly $(\mathrm{p}<0.001)$ lower in PCOS compared to controls. But no significant difference was observed in SD2 ( $>>0.05)$ when compared between the PCOS and control group. In addition, in group PCOS, mean values of plasma catalase $(\mathrm{p}<0.01)$ was significantly lower and plasma MDA level $(\mathrm{p}<0.001)$ was significantly higher than that of control group (Table II).

On correlation analysis, SD1 was negatively and SD1/SD2 ratio was positively correlated with plasma catalase levels but both were statistically non significant $(\mathrm{p}>0.05)$ in PCOS patients (Table III). In addition, SD1 and SD1/SD2 ratio were negatively correlated with plasma MDA level but were statistically non significant $(\mathrm{p}>0.05)$ in these group of patients (Table III). 
Table I: Age, BMI and WHR in two groups $(\mathrm{N}=60)$

\begin{tabular}{lccc}
\hline Variables & $\begin{array}{c}\text { PCOS } \\
(\mathrm{n}=30)\end{array}$ & $\begin{array}{c}\text { Control } \\
(\mathrm{n}=30)\end{array}$ & p value \\
\hline Age & $26.27 \pm 4.29$ & $27.93 \pm 3.92$ & 0.141 \\
(Year) & $(20-35)$ & $(22-33)$ & \\
BMI & $29.65 \pm 3.50$ & $29.00 \pm 2.54$ & 0.415 \\
$\left(\mathrm{Kg} / \mathrm{m}^{2}\right)$ & $(25.09-38.28)$ & $(25.38-34.58)$ & \\
WHR & $0.88 \pm 0.07$ & $0.86 \pm 0.05$ & 0.168 \\
& $(0.75-1.10)$ & $(0.76-0.96)$ & \\
\hline
\end{tabular}

Data were expressed as Mean \pm SD. Values in parentheses indicate ranges; Statistical analysis was done by Independent sample t-test; BMI- Body Mass Index; WHR- Waist Hip Ratio; PCOS - Polycystic ovary syndrome; $\mathrm{n}$ - Number of subjects in each group, $\mathrm{N}=$ Total number of subjects.

Table II: Resting cardiovascular parameters, HRV Poincaré measures and oxidant and antioxidant in two groups $(\mathrm{N}=60)$

\begin{tabular}{lccc}
\hline Variables & PCOS $(\mathrm{n}=30)$ & Control $(\mathrm{n}=30)$ & $\mathrm{p}$ value \\
\hline Pulse rate & $83.47 \pm 7.06$ & $74.97 \pm 7.85$ & $0.000^{* * *}$ \\
(beats $/ \mathrm{min})$ & $(76-102)$ & $(62-90)$ & $0.002^{* *}$ \\
SBP & $121.27 \pm 7.80$ & $115.07 \pm 6.80$ & \\
(mmHg) & $(110-135)$ & $(100-125)$ & $0.004^{* *}$ \\
DBP & $79.73 \pm 5.09$ & $75.63 \pm 5.47$ & \\
(mmHg) & $(70-88)$ & $(65-88)$ & $0.000^{* * *}$ \\
SD1 & $16.92 \pm 4.69$ & $26.77 \pm 10.48$ & \\
(ms) & $(8.63-25.83)$ & $(11.76-61.08)$ & 0.285 \\
SD2 & $54.24 \pm 15.97$ & $58.84 \pm 17.02$ & \\
(ms) & $(21.73-99.63)$ & $(17.77-115.80)$ & $0.000^{* * *}$ \\
SD1/SD2 & $0.32 \pm 0.09$ & $0.46 \pm 0.14$ & \\
ratio & $(0.19-0.58)$ & $(0.23-0.96)$ & $0.006^{* *}$ \\
Plasma catalase & $170.53 \pm 67.69$ & $224.90 \pm 80.11$ & \\
(U/ml) & $(79.00-282.00)$ & $(108.00-389.00)$ & $0.000^{* * *}$ \\
Plasma MDA & $532.94 \pm 234.29$ & $204.55 \pm 96.82$ & $(82.04-478.8)$ \\
(ng/ml) & $(101.3-952.5)$ & & \\
\hline & & & \\
\hline
\end{tabular}

Data were expressed as Mean \pm SD. Values in parentheses indicate ranges; Statistical analysis was done by Independent sample t-test; SBP- systolic blood pressure; DBP- diastolic blood pressure;SD1- Standard deviation of short term RR interval variability; SD2- Standard deviation of Long term RR interval variability; SD1/SD2 ratio-Ratio of short term and long term RR interval variability; MDA- Malondialdehyde; PCOS - Polycystic ovary syndrome; $\mathrm{n}$ - Number of subjects; $* * * \mathrm{p}<0.001 ; * * \mathrm{p}<0.01, \mathrm{~N}=$ Total number of subjects. 
Table III: Correlations of Poincaré HRV measures with plasma catalase and MDA levels in PCOS $(\mathrm{n}=30)$

\begin{tabular}{lccccc}
\hline Variables & \multicolumn{3}{c}{ Catalase } & & \multicolumn{2}{c}{ MDA } \\
\cline { 2 - 3 } \cline { 6 - 6 } & rvalue & $\mathrm{p}$ value & & rvalue & $\mathrm{p}$ value \\
\hline $\mathrm{SD} 1(\mathrm{~ms})$ & -0.060 & 0.752 & & 0.220 & 0.24 \\
$\mathrm{SD} 1 / \mathrm{SD} 2$ ratio & 0.014 & 0.940 & & -0.044 & 0.816 \\
\hline
\end{tabular}

Statistical analysis was done by Pearson's correlation coefficient test; PCOS- Polycystic ovary syndrome MDA- Malondialdehyde n- Number of subjects; SD1- Standard deviation of short term RR interval variability; $\mathrm{SD} 1 / \mathrm{SD} 2$ ratio- Ratio of short term and long term RR interval variability.

\section{Discussion}

The present study assessed the relationship between CANF and oxidative stress in newly diagnosed PCOS patients aged 20-35 years. Age, BMI and WHR in both groups agrees to the observations in similar studies. ${ }^{5,7,17-18}$ In this study, association of higher resting pulse rate, SBP and DBP with PCOS patients are similar to previous studies. ${ }^{6-7}$ These results indicate more deviation of autonomic tonic activity in PCOS from normal.

Analysis of Poincare plot evaluates the sympathovagal balance and observing the beatto-beat HRV. This analysis provides three indices: the standard deviation of the short term RR interval variability (minor axis of the cloud, SD1), the standard deviation of the long term RR interval variability (major axis of the cloud, SD2) and SD1/SD2 represents the ratio of short term and long term variability However, this plot may display abnormalities that are not detectable with traditional time and frequency domain measures easily. ${ }^{19}$ In this study, significant decrement in $\mathrm{SD} 1$ and SD1/SD2 ratio and no significant changes in SD2 indicates association of low parasympathetic activity with PCOS patients. Similar decrement of SD1 was noticed by Saranya and his collaeges. ${ }^{16}$

In the present study, significantly lower plasma catalase levels in PCOS patients suggesting a lower anti-oxidant status in these series of patients agree with other investigators reporting similar observations. ${ }^{11,20-23}$ In addition, plasma MDA level was significantly higher in PCOS patients than that of controls resembling to the observations in similar studies. ${ }^{13,21,22,24}$ These results demonstrate presence of higher oxidative stress in the current series of PCOS patients. Research evidence suggested that obesity, insulin resistance and hyperglycemia contribute to an increase oxidative stress in PCOS.

Oxidative stress affects rostral ventrolateral medulla (RVLM) neurons causing sympatho excitation via interactions with NO. NO exerts a tonic inhibition of central SNS activity by releasing GABA in the RVLM and it also facilitates baroreceptor mediated vagal tone ${ }^{21-23}$. ROS interacts with $\mathrm{NO}$, therefore decreased $\mathrm{NO}$ availability in the RVLM could result in sympathetic over activity and decrease parasympathetic activity ${ }^{25-27}$.

On correlation analysis, SD1 showed non significant negative correlation with plasma catalase and plasma MDA levels in PCOS patients. In addition SD1/SD2 ratio showed non significant positive correlation with plasma catalase and negative correlation with plasma MDA levels in these group of patients.

So, the correlation analysis of this study did not present significant relationship between CANF and oxidative stress in patients with PCOS. However, no mechanism of our research findings could be proposed as no previous study on CANF and oxidative stress in PCOS was found to compare our result.

Volume 16 No. 2 December 2021: 88-94 


\section{Conclusion}

Based on the results of this study it is concluded that autonomic dysfunction and oxidative stress is associated with PCOS but autonomic dysfunction may not be related to oxidative stress in PCOS.

\section{Conflict of interest None}

\section{References}

1. Bozdag G, Mumusoglu S, ZenginD, Karabulut E, Okan Y. The prevalence and phenotypic features of polycystic ovary syndrome: a systematic review and meta-analysis. Hum Reprod 2016; 31(12): 2841-55. DOI: 10.1093/humrep/dew218

2. Rotterdam ESHRE/ASRM-Sponsored PCOS consensus workshop group. Revised 2003 consensus on diagnostic criteria and long-term health risks related to polycystic ovary syndrome (PCOS). Hum Reprod 2004; 19(1): 41-7. DOI: 10.1093/humrep/ deh098

3. Gambineri A, Pelusi C, Vicennati V, Pagotto U, Pasquali R. Obesity and the polycystic ovary syndrome. Int. J. Obes 2002; 26(7): 883-96. DOI: $10.1038=$ sj. $. \mathrm{ijo}=0801994$

4. Gui J, Wang R-H. Cardiovascular autonomic dysfunction in women with polycystic ovary syndrome: a systematic review and meta-analysis. RBM online 2017; 35: 113-20. http://dx.doi.org/ 10.1016/j.rbmo.2017.03.018

5. Kilit C, Kilit TP. Heart rate variability in normal weight patients with polycystic ovary syndrome. Anatol J Cardiol 2017; 17(5):404-09. DOI: 10.14744/ Anatol J Cardiol.2017.7473

6. Hasim ZH, Hamdan FB, Al-Salihi AR. Autonomic dysfunction in women with polycystic ovary syndrome. Iran J Reprod Med 2015; 13(1): 27-34.

7. Saranya K, Pal GK, Habeebullah S, Pal P. Assessment of cardiovascular autonomic function in patients with polycystic ovary syndrome. J Obstet Gynaecol Res P 2014; 40(1): 192-99. http://doi.org/10.1111/ jog. 12154

8. Task Force of the European Society of Cardiology and the North American Society of pacing and electrophysiology. Heart Rate Variability: Standards of Measurement, Physiological Interpretation and Clinical Use. Circulation 1996; 93: 1043-65.
9. Ozkececi G, Unlu BS, Dursun H, Akci O, Koken G, Onrat E, Avsar A. Heart rate variability and heart rate turbulence in patients with polycystic ovary syndrome. Anatol J Cardiol 2016; 16(5): 32327.DOI:10.5152/AnatolJCardiol.2015.6307

10. Lee JY, Baw CK, Gupta S, Aziz N, Agarwal A. Role of oxidative stress in polycystic ovary syndrome. Curr Women's Health Rev 2010; 6(2): 96-107.

11. Santos ACSD, Azevedo GDA, Lemos TMAM. The Influence of oxidative stress in inflammatory process and insulin resistance in obese women with Polycystic Ovary Syndrome. Transl Biomed 2016; 7(4): 1-6. DOI: 10.21767/2172-0479.100100

12. Shirsath A, Aundhakar N, Kamble P. Study of oxidative stress and antioxidant levels in polycystic ovarian disease. Int J Med Biomed Res 2015; 3(4): 16-24.

13. Desai V, Prasad NR, Manohar SM, Sachan A, Narasimha SRPVL, Bitla ARR. Oxidative Stress in Non-Obese Women with Polycystic Ovarian Syndrome. J ClinDiagn Res 2014; 8(7): CC01CC03.DOI: $10.7860 / \mathrm{JCDR} / 2014 / 8125.4530$

14. Fenkci V, Fenkci S, Yilmazer M, Serteser M. Decreased total antioxidant status and increased oxidative stress in women with polycystic ovary syndrome may contribute to the risk of cardiovascular disease. FertilSteril 2003; 80(1): 123-27. DOI:10.1016/S0015-0282(03)

15. Sabuncu T, Vural T, Harma M, Harma M. Oxidative stress in polycystic ovary syndrome and its contribution to the risk of cardiovascualr disease. Clin Biochem 2001; 34(5): 407-13.

16. Saranya K, Pal GK, Habeebullah S, Pal P. Analysis of Poincare plot of heart rate variability in the assessment of autonomic dysfunction in patients with polycystic ovary syndrome. Int J Clin Exp Physiol 2015; 2(1): 34-9. DOI: 10.4103/23488093.155516

17. Yildirir A, Aybar F, Kabakci G, Yarali H, Oto A. Heart rate variability in young women with polycystic ovary syndrome. Ann Noninvasive Electrocardiol 2006; 11(4): 306-12.

18. Hsu C-H, Tsai M-Y, Huang G-S, Lin T-C, Chen KP, Ho S-T, Shyu L-Y, Li C-Y. Acta Anaesthesiologica Taiwanica 2012; 50: 12-18. DOI:10.1016/ j.aat.2012. 03.002

19. Tekin G, Tekin A, Kilic, arslan EB, Haydardedeoglu B, Katircibasi T, Kocum T, Erol T, Colkesen Y, 
Sezgin AT, Muderrisoglu H. Altered autonomic neural control of the cardiovascular system in patients with polycystic ovary syndrome. Int J Cardiol 2008; 130(1): 49-55. DOI:10.1016/j.ijcard.2007.08.037

20. Rasool M, Ahmad R, Rizwan R, Malik A, Asif M, Zaheer A, Jabbar A, Zain M, Qaisrani MM, Qaisrani TB, Mehmood A, Malik IR, Helmi N, Zeyadi M, Choudhry H, Harlev A, Agarwal A, Jamal MS. Interrelationship of circulating biochemical markers of oxidative stress and comorbid condition in polycystic ovary syndrome. Biomed Res 2018; 29(21): 3779-83.

21. Özer A, Bakacak M, Kiran H, ErcanÖ, KöstüB, Pektap MK, Kýlýnç M, Aslan F. Increased oxidative stress is associated with insulin resistance and infertility in polycystic ovary syndrome. Ginekologia Polska 2016; 87(11): 733-38. DOI: 10.5603/GP.2016.0079

22. Kandasamy S, Sivagamasundari RI, Bupathy A, Sethubathy S, Gobal V. Evaluation of insulin resistance and oxidative stress in obese patients with polycystic ovary syndrome. IJABPT 2010; 1(2): 391-98.
23. Mohan SK, Priya V. Lipid peroxidation, glutathione, ascorbic acid, vitamin E, antioxidant enzyme and serum homocysteine status in patients with polycystic ovary syndrome. Biol Med 2009; 1(3): 44-9.

24. Kuscu NK, Var A. Oxidative stress but not endothelial dysfunction exists in non-obese, young group of patients with polycystic ovary syndrome. Acta Obstet Gynecol Scand 2009; 88(5): 612-17. DOI: $10.1080 / 00016340902859315$

25. Salim S. Oxidative Stress and the Central Nervous System. J Pharmacol Exp Ther 2017; 360: 20105. http://dx.doi.org/10.1124/jpet.116.237503

26. Campese VM, Ye S, Zhong H, Yanamadala V, Ye Z, Chiu J. Reactive oxygen species stimulates central and peripheral sympathetic nervous activity. Am J Physiol Heart Circ Physiol 2004; 286: 695-03.

27. Kishi. Regulation of the sympathetic nervous system by nitric oxide and oxidative stress in the rostral ventrolateral medulla: 2012 Academic Conference Award from the Japanese Society of Hypertension. Hypertens Res 2013; 36: 845-51. DOI:10.1038/ hr.2013.73 